\title{
The expression of interleukin-25 increases in human coronary artery disease and is associated with the severity of coronary stenosis
}

\author{
(D) Yao Xu", (D) Jing Ye\#, (D) Menglong Wang\#, (D) Jianfang Liu, (D) Zhen Wang, \\ (D) Huimin Jiang, (D) Di Ye, (D) Jishou Zhang, (D) Jun Wan \\ Department of Cardiology, Renmin Hospital of Wuhan University; Cardiovascular Research Institute, \\ Wuhan University, Hubei Key Laboratory of Cardiology; Wuhan-China
}

\section{ABSTRACT}

Objective: Interleukin (IL) 25, also known as IL-17E, is an inflammatory cytokine and has been demonstrated to be closely related to cardiovascular diseases by regulating immunity and inflammation, including atherosclerosis. This study was aimed to evaluate the expression of IL-25 in patients with coronary artery disease (CAD).

Methods: In this study, the expression of IL-25 in normal $(n=6)$ and atherosclerotic $(n=10)$ human coronary arteries was detected by immunofluorescent staining. In addition, the serum IL-25, IL-6, and tumor necrosis factor (TNF) a concentrations in stable angina pectoris (SAP, $n=44$ ), unstable angina pectoris (UAP, $n=46$ ), acute myocardial infarction ( $A M I, n=34)$, and non-CAD (control, $n=36$ ) were measured using enzyme-linked immunosorbent assay (ELISA) kits.

Results: IL-25 was significantly increased in coronary arteries of CAD patients when compared with normal coronary arteries, with macrophages and T lymphocytes being the sources of IL-25, especially macrophages. Moreover, the serum concentrations of IL-25 were markedly elevated in CAD patients and gradually increased in SAP, UAP, and AMI groups. In addition, IL-25 levels were positively correlated with the IL-6 and TNF-a levels, and Gensini score in CAD patients. Logistic regression analysis showed that IL-25 was independently positively correlated with the occurrence of acute coronary syndrome (ACS). A receiver operator characteristic curve suggested that IL-25 presented a significant diagnosis value in ACS.

Conclusion: IL-25 is increased in the coronary arteries and serum of CAD patients and is associated with the severity of coronary stenosis and the occurrence of ACS, suggesting that IL-25 may be one of the biomarkers of ACS. (Anatol J Cardiol 2020; 23: 151-9)

Keywords: interleukin 25, coronary artery disease, inflammation, Gensini score

\section{Introduction}

Coronary artery disease (CAD) is one of the most fatal cardiovascular diseases with a high hospitalization rate and mortality (1). Inflammation has been identified to participate in CAD partly by acting on atherosclerosis (2). Based on the critical role of various inflammatory factors and mediators in the pathophysiological processes of atherosclerosis, there are increasing numbers of investigations focused on novel and efficient therapies to ameliorate the immune and inflammatory response of atherosclerosis (3-6).

The interleukin (IL) 17 cytokine family, including IL-17A, IL17B, IL-17C, IL-17D, IL-17E (also known as IL-25), and IL-17F, was found to be involved in inflammatory responses $(7,8)$. Growing evidence showed that the IL-17 cytokine family played important roles in cardiovascular diseases. For instance, IL-17A was increased in the plasma of unstable angina and acute myocardial infarction patients, and no significance was noted between patients with complex and simple lesions $(9,10)$. Animal studies also showed that IL-17A is increased in the left ventricle infarcted region of rats after myocardial infarction. Moreover, endothelial cells in the infarcted region were observed to stain positively for IL-17A, which suggested that IL-17A might be involved in cardiac repair and inflammation (11). Liao et al. (12) reported that IL-17A might have the capacity to regulate cardiomyocyte apoptosis and neutrophil infiltration to participate in myocardial ischemia/reperfusion injury (13).

"These authors contributed equally to this work.

Address for correspondence: Jun Wan, MD, Department of Cardiology, Renmin Hospital of Wuhan University; Cardiovascular Research Institute, Wuhan University; Hubei Key Laboratory of Cardiology, 430060, Wuhan-China

Phone: +86-27-88041911 Fax:+86-27-88042293 E-mail: wanjun@whu.edu.cn - whuwanjun@163.com

Accepted Date: 07.11.2019 Available Online Date: 13.02.2020

(C) Copyright 2020 by Turkish Society of Cardiology - Available online at www.anatoljcardiol.com DOI:10.14744/AnatolJCardiol.2019.24265 
IL-25 is also a member of the IL-17 cytokine family, which possesses unique structures and has the capacity to regulate immune and inflammatory responses (14) and angiogenesis (15). Further evidence showed that IL-25 promotes cell proliferation, inhibits apoptosis (16), and affects the development of fibrosis and hypoxic ischemic injury (17), tumor metastasis (18) and metabolism processes $(19,20)$. Experiments using IL-25 for the treatment of atherosclerosis-prone apolipoprotein (apo) E-deficient mice showed its ability to protect against atherosclerosis without affecting the adaptive Th2 responses (21). However, the IL-25 levels in patients with CAD are still unclear. In this study, we detected the serum and coronary artery tissue expression of IL-25 in CAD and attempted to explore the possible clinical associations between IL-25 and CAD.

\section{Methods}

\section{Collection of coronary artery tissues}

This study protocol was approved by the Medical Ethics Committee of the Renmin Hospital of Wuhan University. All donors or their families signed an informed consent form before tissue harvesting. Human coronary artery tissue with atherosclerotic plaques $(n=10)$ was obtained from CAD patients undergoing heart transplantation, whereas control arteries $(n=6)$ were obtained from normal donors who failed to match for a transplant recipient. All donors in whom other diseases were reported to affect the expression of IL-25 including allergic diseases, autoimmune diseases, and tumor were ruled out. The tissues were fixed in formalin at the end of the surgery and then embedded in paraffin for immunofluorescence.

\section{Immunofluorescence}

After the tissue samples were fixed in formalin, coronary artery tissues embedded in paraffin were cut into $5 \mu \mathrm{m}$ slices. After washing in PBS, the sections were subsequently sealed with $8 \%$ sheep serum for $1 \mathrm{~h}$ and incubated at $4^{\circ} \mathrm{C}$ overnight with primary antibodies. Immunofluorescent staining was used for IL-25 expression by anti-IL-25 antibodies (R\&D Systems, USA). Double immunofluorescent staining was used to detect the source of IL-25 by anti-CD4 antibodies (Biolegend, USA), anti-CD68 antibodies (Abcam, UK), and anti-a- smooth-muscle actin (SMA) antibodies (Abcam, UK). Then, the sections were washed with PBS followed by a secondary antibody incubation at $37^{\circ} \mathrm{C}$ for 1 h. The nuclei were stained with 4',6-diamidino-2-phenylindole (R\&D Systems, USA). Finally, the sections were visualized by fluorescence microscopy.

\section{Collection and conservancy of human blood samples}

Patients who suffered from chest pain and had undergone coronary angiography were recruited from January 2018 to March 2018 in the Renmin Hospital of Wuhan University. The study protocol was approved by the Medical Ethics Committee of the Renmin Hospital of Wuhan University. The exclusion crite- ria for this study included patients who were too young or too old $(<20$ or $>80$ years old) $(n=5)$ and those with severe liver damage, severe renal dysfunction, and a prior history of drug assumption $(n=2)(22)$. Meanwhile, all donors in whom other diseases were reported to affect the expression of IL-25 including allergic diseases, autoimmune diseases, and tumor were ruled out.

According to the diagnoses, 37 patients were assigned to the non-CAD group (control group), 44 patients to the stable angina pectoris (SAP) group, 48 patients to the unstable angina pectoris (UAP) group, and 34 patients to the acute myocardial infarction (AMI) group. The CAD patients group included the SAP, UAP, and AMI groups. Vacutainers coated with sodium heparin were used to collect blood samples upon admission. Thirty-four AMI patients underwent reperfusion therapy after blood collection. Serum was isolated after centrifugation for 20 min at 4000 $\mathrm{g}$ within $1 \mathrm{~h}$ of collection, aliquoted, and stored at $-80^{\circ} \mathrm{C}$ until analysis. In addition, patients with blood sample hemolysis $(n=1)$ in the control group and lipaemia $(n=2)$ in the UAP group were also excluded from this study. All of the donors or their families have provided an informed consent.

\section{Measurement of serum IL-25, IL-6, and TNF- $\alpha$ levels}

Serum concentrations of IL-25 (R\&D Systems, USA), IL-6, and TNF-a (both from eBioscience, USA) were measured using the enzyme-linked immunosorbent assay (ELISA) kits according to the manufacturer's instructions.

\section{Gensini score}

The Gensini score was used to estimate the severity of coronary stenosis according to the results of a coronary angiography as previously reported (22). The coronary angiography was conducted by the attending practicing physicians. Then, we recorded the subjects' Gensini scores according to the results of the coronary angiography.

\section{Echocardiography measurement}

The patients' left ventricular ejection fraction (LVEF) was measured using a VIVID E9 (GE Healthcare, USA) ultrasonography machine as previously reported (22). The LVEF values were recorded for all subjects.

\section{Statistical analysis}

The statistical software package SPSS 19.0 was used for data analysis. All data were tested for normality using the KolmogorovSmirnov test. Clinical characteristics with normal distribution were presented as mean \pm standard deviation. Student's t-tests were performed when two normal distribution groups were compared and one-way analysis of variance (ANOVA) was used to test the difference between multiple groups. Data without normal distribution were presented as the median (minimum-maximum) and compared by means of the Mann-Whitney $U$ test. The correlations in continuous variables with normal distribution were evaluated by Pearson correlation analysis. Spearman's correlation analysis 
was used in continuous variables without normal distribution. Simple linear regression analyzes and subsequent binary logistic regression analyzes were used to identify whether IL-25 was an independent predictor of ACS. A receiver operator characteristic (ROC) curve was used to evaluate the diagnosis value of cytokines in ACS. $P<0.05$ was considered statistically significant.

\section{Results}

\section{Basic clinical characteristics of patients who provided coronary artery tissues}

From the basic clinical characteristics of the patients who provided coronary artery tissues, several significant differences were found between control and CAD patients, including higher levels of TC and LDL-C, but lower LVEF in CAD patients. However, no difference was observed in other clinical characteristics, including gender, age, smoking, hypertension, BMI, SBP, DBP, Glu, TG, HDL-C, eGFR, CRP, and time. These clinical characteristics of patients are shown in Table 1.

\section{Expression of IL-25 in human atherosclerotic plaques}

To determine the expression of IL-25 in human atherosclerotic plaques, immunofluorescent staining was performed. We

\begin{tabular}{|c|c|c|}
\hline Characteristics & Control & CAD \\
\hline Gender (M/F) & $3 / 3$ & $6 / 4$ \\
\hline Age (years) & $62.0(46.0-76.0)$ & $62.5(48.0-73.0)$ \\
\hline Smoking (\%) & 50 & 50 \\
\hline Hypertension (\%) & 50 & 70 \\
\hline BMI $\left(k g / m^{2}\right)$ & $20.6(17.9-23.6)$ & $21.1(18.8-24.5)$ \\
\hline $\mathrm{SBP}(\mathrm{mm} \mathrm{Hg})$ & $125.5(118.0-136.0)$ & $118.0(102.0-137.0)$ \\
\hline $\mathrm{DBP}(\mathrm{mm} \mathrm{Hg})$ & $78.5(66.0-87.0)$ & $88.5(68.0-99.0)$ \\
\hline Glu (mmol/L) & $5.6(3.8-6.5)$ & $5.95(4.9-7.1)$ \\
\hline $\mathrm{TG}$ (mmol/L) & $1.4(0.7-1.9)$ & $1.9(0.9-2.3)$ \\
\hline $\mathrm{TC}(\mathrm{mmol} / \mathrm{L})$ & $3.4(3.0-4.6)$ & $4.0(3.4-6.0)^{*}$ \\
\hline HDL-C (mmol/L) & $1.40(0.88-1.90)$ & $1.4(0.9-1.8)$ \\
\hline LDL-C (mmol/L) & $2.2(1.9-3.3)$ & $3.5(2.0-4.5)^{*}$ \\
\hline eGFR (mL/min) & $92.0(80.0-100.0)$ & $87.5(78.0-104.0)$ \\
\hline $\mathrm{CRP}$ (mg/L) & $3.0(0.44-4.5)$ & $3.9(2.0-4.5)$ \\
\hline LVEF (\%) & $56.0(50.0-60.0)$ & $31.0(24.0-36.0)^{*}$ \\
\hline Time (h) & $3.4(2.9-4.0)$ & $3.1(2.5-4.2)$ \\
\hline
\end{tabular}

${ }^{*} P<0.05$ vs. control. BMI - body mass index; CRP - C-reactive protein; DBP - diastolic blood pressure; eGFR - estimated glomerular filtration rate; Glu - fasting glucose;

HDL-C - high-density lipoprotein cholesterol; LDL-C - low-density lipoprotein

cholesterol; LVEF - left ventricular ejection fraction; SBP - systolic blood pressure;

TC - total cholesterol; TG - total triglycerides; Time - time intervals between the beginning of surgery and collection of tissue sampling; CAD - coronary artery disease; $\mathrm{M}$ - male; F - female found that IL-25 was highly expressed in coronary arteries with atherosclerotic plaques but lowly expressed in normal arteries (Fig. 1a). Specific antibodies, such as human anti-CD4 antibodies, anti-CD68 antibodies, and anti-a-SMA antibodies, were used to show markers for T-helper cells, macrophages, and smooth muscle cells, respectively. In addition, immunofluorescent double staining displayed that IL-25 was almost not expressed by smooth muscle cells in atherosclerotic arteries but was expressed by macrophages and T lymphocytes, especially the macrophages (Fig. 1b).

\section{Basic clinical characteristics of patients who provided blood samples}

Serum levels of Glu, LDL-C, and cardiac troponin I (TnI), as well as the percent of smoking and obesity, SBP, DBP, CRP, and the Gensini score were significantly higher in CAD groups when compared to those of the control group. However, there were no significant differences between the control and the CAD groups in terms of other clinical parameters including diabetes and hyperlipidemia, age, HbAlc, TG, TC, HDL-L, eGFR, ALT, LVEF, NT-proBNP, and oral hypoglycemia. The basic clinical characteristics of each group are presented in Table 2.

\section{Serum IL-25, IL-6, and TNF-a levels in the four groups}

According to the results of the ELISA, IL-25 levels were significantly higher in the CAD patients compared to those in the control group (Table 3) and gradually increased in the SAP, UAP, and AMI groups (150.7 pg/mL vs. $379.8 \mathrm{pg} / \mathrm{mL}$ vs. $484.7 \mathrm{ng} / \mathrm{mL}$ vs. $634.3 \mathrm{ng} / \mathrm{mL}, \mathrm{p}<0.05$ ) (Fig. 2a). Homoplastic changes were found in IL-6 and TNF-a levels (Fig. $2 b$ and $2 c$ ); however, not all of the differences noted were statistically significant. The serum levels of the various cytokines in the four groups of patients are listed in Table 3.

\section{The correlation between serum cytokine levels and the} Gensini score

Pearson correlation analysis or Spearman's correlation analysis were performed in 124 CAD patients to evaluate the relationship among IL-25, IL-6, and TNF- $a$ and the Gensini score. The results showed that serum levels of IL-25 were markedly positively correlated with IL-6 $(r=0.304, p<0.001)$ and TNF- $a$ $(r=0.403, p<0.001)$. The concentrations of IL-6 were also identified to be positively correlated with TNF- $a(r=0.201, p=0.026)$. Additionally, the concentrations of serum IL-25 ( $r=0.495, p<0.001)$, IL-6 ( $r=0.440, p<0.001)$, and TNF- $a(r=0.507, p<0.001)$ were positively correlated with the Gensini score (Fig. $3 a-3 c$ ).

\section{The association between serum IL-25 and ACS}

Simple linear regression analysis and binary logistic regression analysis were performed to confirm whether IL-25 is a possible predictor for ACS. The results of the simple linear regression analysis suggested that IL-25, IL-6, TNF-a, cTnI, CRP, Glu, $L D L$, and SBP levels showed a potential $(p<0.05)$ correlation 

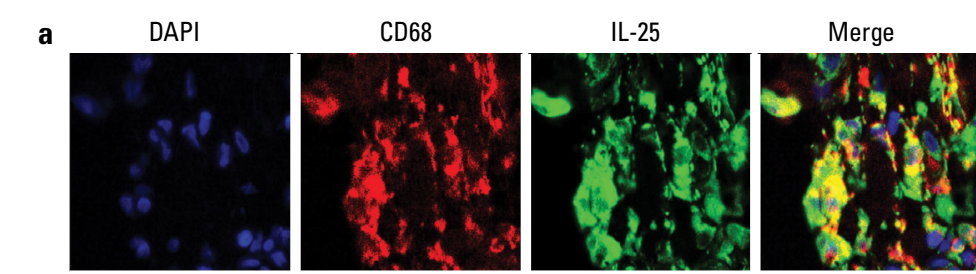

DAPI
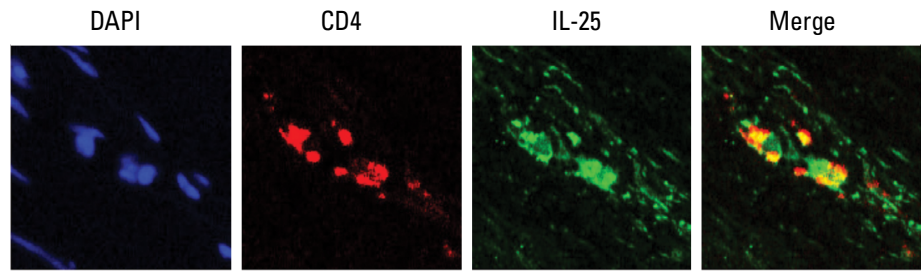

IL-25

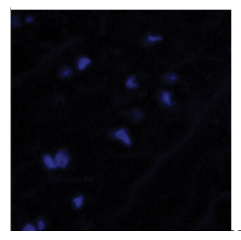

a-SMA

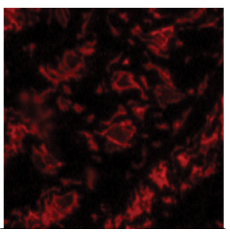

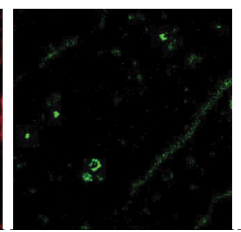
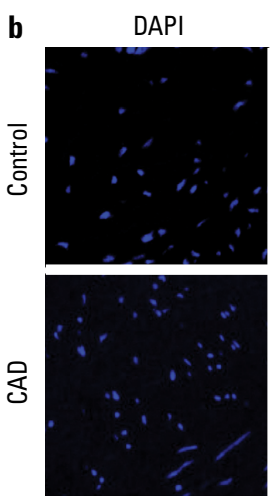
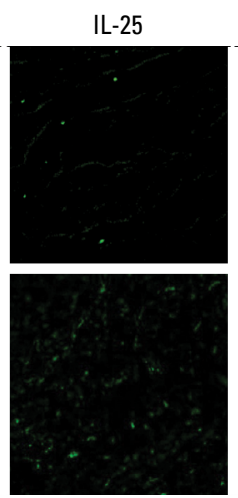
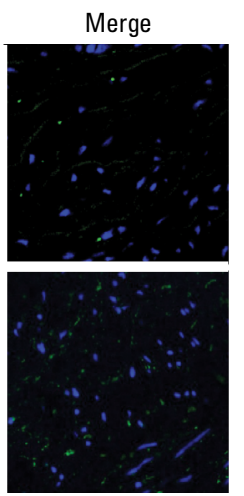

Figure 1. IL-25 expression in human atherosclerotic plaques. (a) IL-25 levels in normal and CAD coronary artery tissues were measured by immunofluorescent staining $(200 \times)$. (b) Double staining for IL-25 expression, Th cells, macrophages, and smooth muscle cells in human atherosclerotic plaques $(400 \times)$

CAD - coronary artery disease

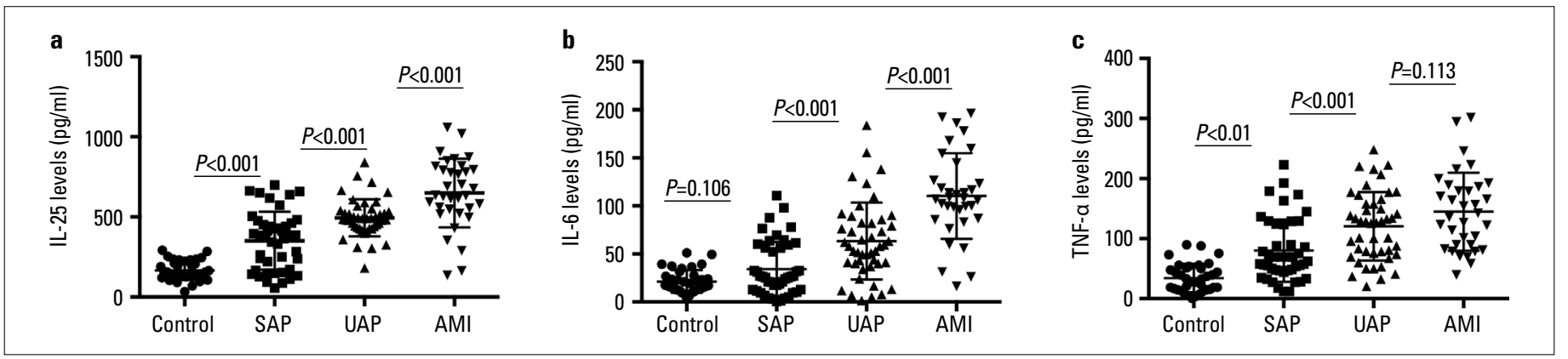

Figure 2. Serum IL-25, IL-6, and TNF-a levels in the four groups. (a) Serum IL-25 levels in patients with SAP, UAP, AMI, and control groups were measured. (b) Serum IL-6 levels were measured in the four groups. (c) Serum TNF-a levels were measured in each group

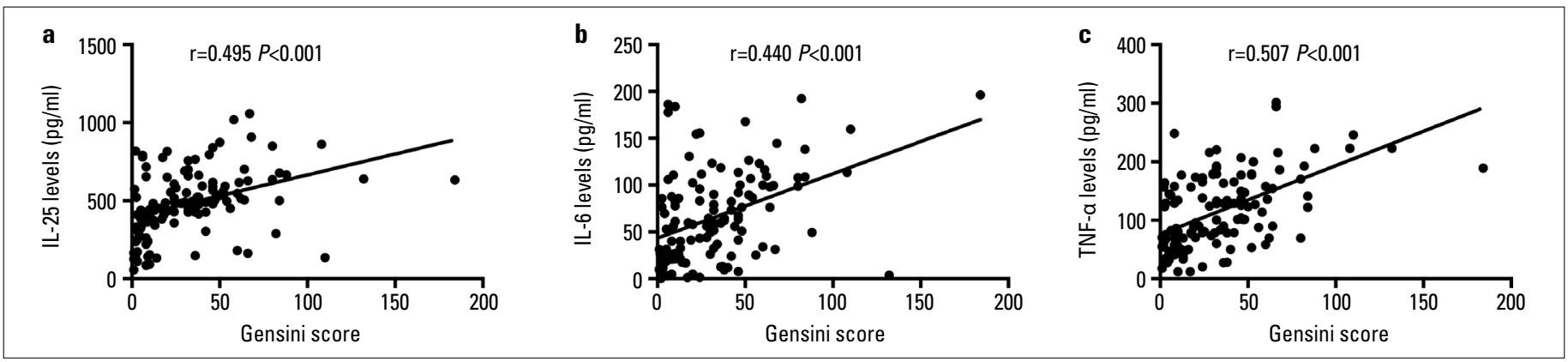

Figure 3. Spearman's correlation of circulating cytokines and Gensini score. Correlation among (a) IL-25 levels, (b) IL-6 levels, (c) TNF-a levels, and Gensini score

with the onset of ACS whereas smoking, DBP, BMI, HbAlc, TC, age, and gender showed no obvious trends $(p>0.05)$ with the onset of ACS. Furthermore, IL-25, IL-6, TNF-a, cTnl, CRP, Glu, LDL, and SBP levels were used to perform binary logistic regression analysis. The further results showed that serum levels of IL-25 (OR 1.006, 95\% Cl 1.002 to $1.011 ; p=0.004$ ), IL-6 (OR 1.036, 95\% Cl 
Table 2. Information of clinical characteristics of patients who provided blood samples

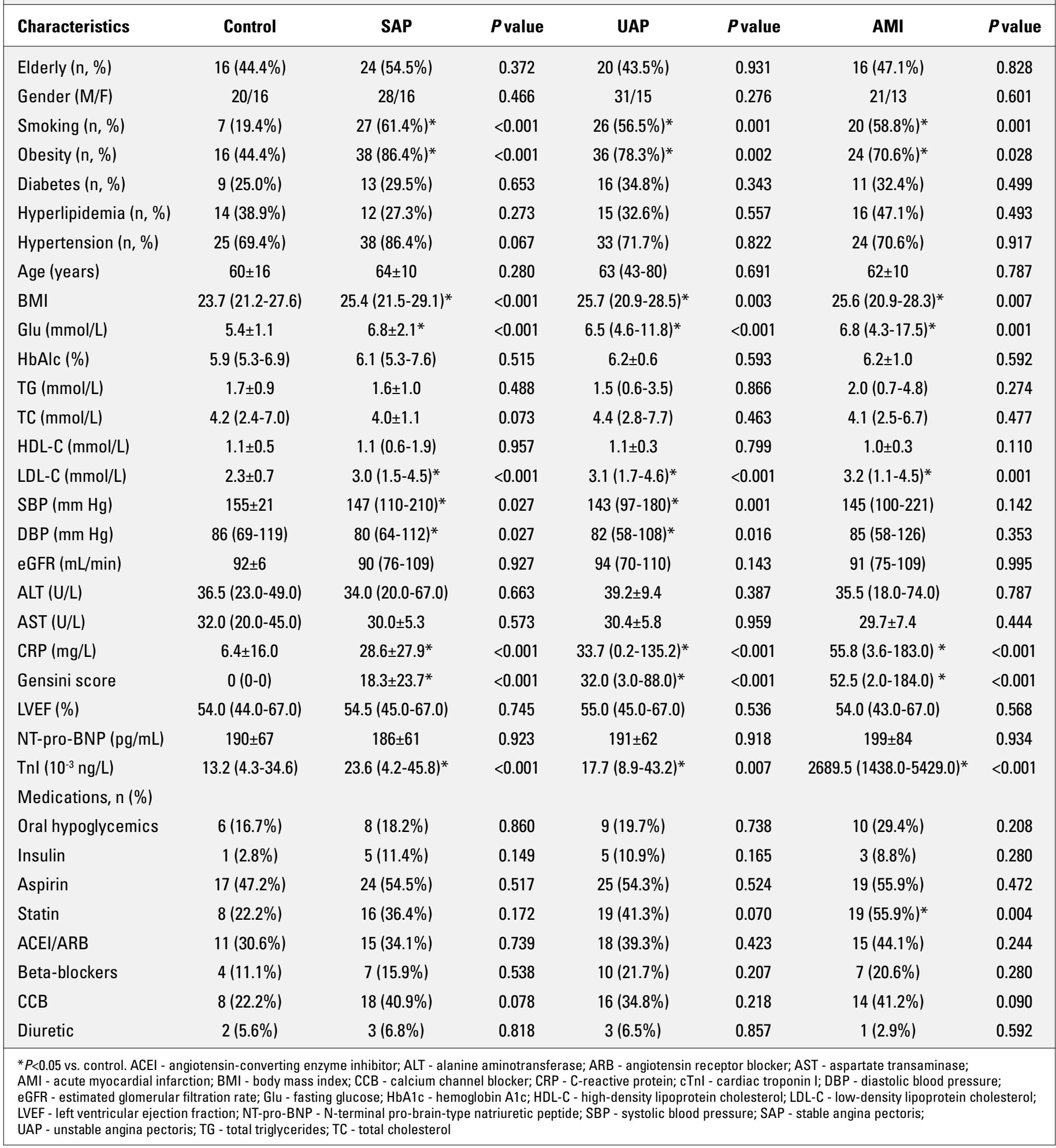

1.017 to $1.055 ; \mathrm{p}<0.001$ ), and Glu (OR 1.471, 95\% $\mathrm{Cl} 1.040$ to 2.081; $\mathrm{p}=0.029$ ) seemed to be independent predictors for ACS (Table 4). In this study, part of variables including TNF-a, cTnl, CRP, LDL, and SBP levels were not the independent predictors for the occurrence of ACS.
The diagnostic value of cytokines and cTnl in ACS

We further applied ROC analysis on diagnostic values of serum cTnl, IL-25, IL-6, and TNF-a concentrations in ACS. As shown in Figure $4 \mathrm{a}$ and $4 \mathrm{~b}$, each of these cytokines and $\mathrm{cTnl}$ seem to have particular diagnostic value for ACS, whereas the 
Table 3. Serum IL-25, IL-6, and TNF-a levels in each group

\begin{tabular}{|c|c|c|c|c|c|c|c|}
\hline & Control & SAP & $P$ value & UAP & $P$ value & AMI & $P$ value \\
\hline IL-25 (pg/mL) & $150.7(33.2-292.4)$ & $379.8(57.3-699.3)^{*}$ & $<0.001$ & $484.7(180.9-841.0)^{*}$ & $<0.001$ & $634.3(135.7-1058.0)^{*}$ & $<0.001$ \\
\hline TNF-a (pg/mL) & $30.4(0.6-90.0)$ & $80.0 \pm 51.9^{*}$ & $<0.001$ & $127.3(20.4-248.1)^{*}$ & $<0.001$ & $139.1(39.5-301.5)^{*}$ & $<0.001$ \\
\hline
\end{tabular}

Table 4. Association between cytokines, clinical characteristics, and the presence of acute coronary syndrome assessed by simple linear regression analysis and further multiple linear regression analysis

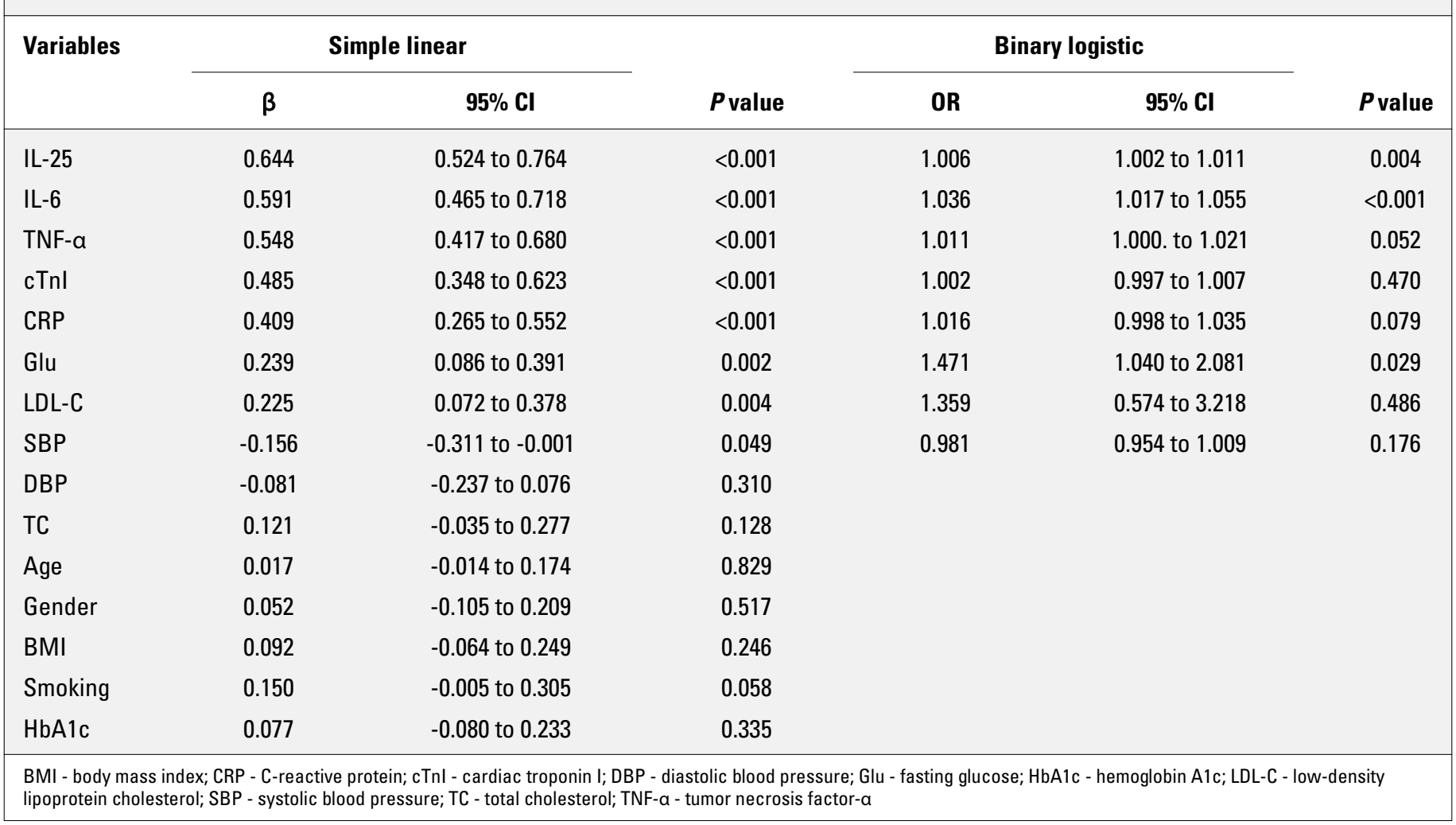

combination of three cytokines presented a bit more preferable diagnostic value than three cytokines individually did in ACS.

\section{Discussion}

In the present study, we found for the first time that IL-25 was increased in human atherosclerotic arteries and that T Iymphocytes and macrophages, especially the macrophages, rather than smooth muscle cells, were the source of IL-25 in atherosclerotic plaques. We also found that IL-25 levels were increased in the serum of CAD patients and positively correlated with the severity of coronary stenosis. Binary logistic regression analysis suggested that IL-25 might independently correlate with the onset of ACS.

Previous studies had demonstrated that IL-25 was associated with the progression of various diseases of the immune system, such as asthma (23), rheumatoid arthritis (24), multiple sclerosis (25) and inflammatory bowel disease (26). However, IL-25 expression in various diseases involving other systems is controversial. Growing evidence has shown that IL-25 is upregulated in serum of patients with allergic diseases such as respiratory diseases exacerbated by aspirin (27) but without significant changes in patients with severe asthma (28) or uncontrolled asthma (29). In addition, Aalberse et al. (30) showed that IL-25 was also highly enhanced in children's plasma response to peanuts. In autoimmune diseases, IL-25 was observed to be elevated in the plasma of rheumatoid arthritis patients and serum of Churg-Strauss syndrome patients compared to healthy individuals $(31,32)$. In our study, the results showed that serum concentrations and atherosclerotic plaques expression of IL-25 in CAD patients were significantly increased and that IL-25 was also positively correlated with the Gensini score, further con- 

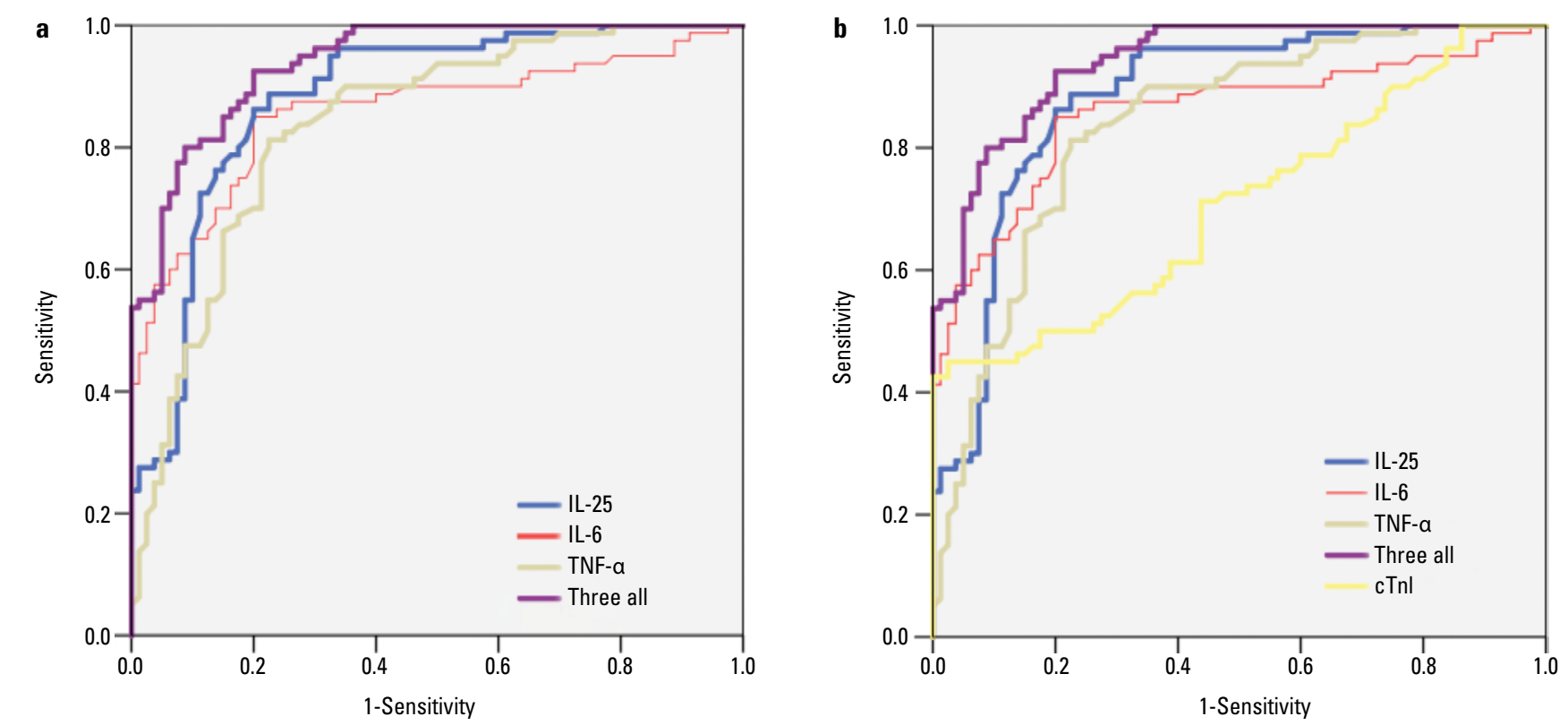

Figure 4. Diagnostic value of cytokines and cTnl in ACS. (a) ROC curve of IL-25, IL-6, TNF-a, and combining the three cytokines for ACS. (b) ROC curve of IL-25, IL- 6 , and TNF-a, combining the three cytokines and cTnl for ACS

firming that IL-25 levels may be closely related with the severity of coronary stenosis. These results suggested that IL-25 might play an important role in the progression of atherosclerosis.

After it was first found to be expressed by Th2-polarized CD4+ T cells, IL-25 has been identified to be extensively produced and to locally or generally participate in inflammatory, infectious, or autoimmune diseases (33-35). IL-25 has been identified to be produced by several different cells, such as bone marrowderived mast cells (36), alveolar macrophages (37), eosinophils, basophils (38), epithelial, (15) and endothelial cells (39). Our results of immunofluorescence double staining were consistent with the above conclusions, and for the first time, we suggest that macrophages and T lymphocyte are important sources of IL-25 in human atherosclerotic plaques. This suggestion is a significant addition to the sources of IL-25 and indicates that IL-25 may influence the development of atherosclerosis via regulation of the inflammatory response.

Atherosclerosis is well known to be an underlying pathophysiological mechanism of CAD and various inflammatory cells and factors have been identified to play important roles in the development of atherosclerosis (40). Clinical, experimental, and animal studies have supported the hypothesis that inflammatory cells are recruited to the intima during the beginning of atherosclerosis and monocytes further differentiate into tissue macrophages after translocation to the arteries and subsequently become foam cells. In response to the development of an atheroma, one type of macrophages named M1 macrophages have the function of secreting the proinflammatory cytokines such as IL-1 $\beta$ and TNF- $\alpha$, which are involved in the stimulation of IL-6 production (41). Kampits et al. (42) reported that CAD patients who experienced major events had higher concentrations of
TNF- $a$ and IL-6. Min et al. (43) found that serum levels of TNF-a and IL- 6 were significantly more elevated in ACS patients than those in controls and that these levels were associated with high Gensini scores. In our present study, we found that the concentrations of serum TNF- $\alpha$ and IL- 6 are not only increased in CAD patients but also associated with IL-25 levels and Gensini scores, which reflect the severity of coronary stenosis. Our results are consistent with previous findings and further suggested the predictive role of TNF- $a$ and IL-6 in CAD as shown in the Spearman correlation analysis and logistic regression analysis. Therefore, we surmised that IL-25 could affect levels of TNF-a and IL- 6 in CAD patients.

As important members of inflammatory cytokines, IL-6, and TNF- $a$ have been identified to be synthesized by macrophages, $B$ cells, CD4 effector T-helper cells, and some other immune cells (44). As a Th2 cytokine, IL-25 has been reported to regulate several inflammatory and immune cells in the progression of several diseases. For instance, Kleinschek et al. (45) suggested that experimental autoimmune encephalomyelitis IL-25 knockout mice presented increased levels of IL-23 in the periphery and enhanced $\mathrm{T}$ cells in the central nervous system, which produced IL-17, IFN- $\gamma$, and TNF. IL-25 also enhanced the functions of Th2 memory cells through the sustained expression of GATA-3, c-MAF, and JunB independent of IL-4 (38). In contact hypersensitivity induced by fluorescein isothiocyanate, IL-25 secreted by mast cells and nonimmune cells can stimulate dermal dendritic cells to produce IL-1 $\beta$ and contributes to the activation of Th17 instead of Th2 cells (46). Furthermore, with exogenous IL-25 treatment of apoE/Rag-1 deficient mice, splenic innate lymphoid type 2 cells were extensively enhanced and plasma IL-5 was also increased. In addition, apoE- 
deficient mice presented reduced aortic atherosclerosis after injection of recombinant IL-25, which suggests that IL-25 may have a protective role in atherosclerosis (21). IL-25 deficient mice were also used and showed an aggravation of the aortic atherosclerotic plaques with elevated Th1/Th17 proinflammatory response, suggesting a role for IL-25 in the maintenance of the $T$ cells in patients suffering from hyperlipidemias (47). Our studies first found that high serum IL-25 levels were associated with an increased risk of suffering ACS. Data in ROC analysis suggested that IL-25 might have the function of reflecting the stability of plaque. The small sample size of our study might contribute to these results, although further large amount of subjects will be more convincing. These results indicated that inflammatory cytokine IL-25 would be tightly related to atherosclerosis progress, especially unstable plaque, and shows potential to be a biomarker for ACS patients.

\section{Study limitations}

However, there are some limitations in the present study. For instance, oxidative stress, which also plays critical roles in the development of atherosclerosis, might be another mechanism for IL-25 involving in ACS. Therefore, further investigations should concentrate on the relationship between IL-25 and oxidative stress in atherosclerosis. Another limitation of the study is that the number of the patients was too small and more subjects should be included to reduce any margins of error.

\section{Conclusion}

The results of our study identified that IL-25 is highly expressed in atherosclerotic arteries of CAD patients and is mainly produced by macrophages and T lymphocyte cells. Additionally, IL-25 levels in serum were first significantly increased in CAD patients especially in AMI patients and positively correlated with the severity of coronary atherosclerosis. Further analysis identified that high serum IL-25 levels were associated with an increased risk of suffering plaque instability and high predictive value of IL-25 in ACS.

\section{Conflict of interest: None declared.}

Peer-review: Externally peer-reviewed.

Authorship contributions: Concept - Y.X., J.Y., J.W.; Design - Y.X., M.W., H.J.; Supervision - Y.X., J.Y., M.W.; Funding - J.W.; Materials - J.L., D.Y., J.Z.; Data collection and/or processing - J.L., Z.W., H.J.; Analysis and/or interpretation - H.J., D.Y., J.Z.; Literature search - Y.X., J.Y., J.W.; Writing - Y.X., J.Y., M.W.; Critical review - Y.X., J.L., Z.W., D.Y.

\section{References}

1. Roger VL, Go AS, Lloyd-Jones DM, Benjamin EJ, Berry JD, Borden WB, et al.; American Heart Association Statistics Committee and
Stroke Statistics Subcommittee. Executive summary: heart disease and stroke statistics--2012 update: a report from the American Heart Association. Circulation 2012; 125: 188-97. [CrossRef]

2. Christodoulidis G, Vittorio TJ, Fudim M, Lerakis S, Kosmas CE. Inflammation in coronary artery disease. Cardiol Rev 2014; 22: 279-88.

3. Khan R, Spagnoli V, Tardif J, L'Allier PL. Novel anti-inflammatory therapies for the treatment of atherosclerosis. Atherosclerosis 2015; 240: 497-509. [CrossRef]

4. Golia E, Limongelli G, Natale F, Fimiani F, Maddaloni V, Pariggiano I, et al. Inflammation and cardiovascular disease: from pathogenesis to therapeutic target. Curr Atheroscler Rep 2014; 16: 435. [CrossRef]

5. Ridker PM, Everett BM, Thuren T, MacFadyen JG, Chang WH, Ballantyne $C$, et al.; CANTOS Trial Group. Antiinflammatory Therapy with Canakinumab for Atherosclerotic Disease. N Engl J Med 2017; 377: 1119-31. [CrossRef]

6. Ridker PM, MacFadyen JG, Thuren T, Everett BM, Libby P, Glynn RJ; CANTOS Trial Group. Effect of interleukin-1beta inhibition with canakinumab on incident lung cancer in patients with atherosclerosis: exploratory results from a randomised, double-blind, placebo-controlled trial. Lancet 2017; 390: 1833-42. [CrossRef]

7. Kolls JK, Linden A. Interleukin-17 family members and inflammation. Immunity 2004; 21: 467-76. [CrossRef]

8. Gu C, Wu L, Li X. IL-17 family: cytokines, receptors and signaling. Cytokine 2013; 64: 477-85. [CrossRef]

9. Hashmi S, Zeng OT. Role of interleukin-17 and interleukin-17-induced cytokines interleukin- 6 and interleukin-8 in unstable coronary artery disease. Coron Artery Dis 2006; 17: 699-706. [CrossRef]

10. Liang J, Zheng Z, Wang M, Han L, Zheng Z, Peng J, et al. Myeloperoxidase (MPO) and interleukin-17 (IL-17) plasma levels are increased in patients with acute coronary syndromes. $\mathrm{J}$ Int Med Res 2009; 37: 862-6. [CrossRef]

11. Avalos AM, Apablaza FA, Quiroz M, Toledo V, Pena JP, Michea L, et al. IL-17A levels increase in the infarcted region of the left ventricle in a rat model of myocardial infarction. Biol Res 2012; 45: 193-200.

12. Liao YH, Xia N, Zhou SF, Tang TT, Yan XX, Lv BJ, et al. Interleukin17A contributes to myocardial ischemia/reperfusion injury by regulating cardiomyocyte apoptosis and neutrophil infiltration. J Am Coll Cardiol 2012; 59: 420-9. [CrossRef]

13. Harrington RA. Targeting Inflammation in Coronary Artery Disease. N Engl J Med 2017; 377: 1197-8. [CrossRef]

14. Valizadeh A, Khosravi A, Zadeh LJ, Parizad EG. Role of IL-25 in Immunity. J Clin Diagn Res 2015; 9: OE01-4. [CrossRef]

15. Corrigan CJ, Wang W, Meng 0 , Fang C, Wu H, Reay V, et al. T-helper cell type 2 (Th2) memory T cell-potentiating cytokine IL-25 has the potential to promote angiogenesis in asthma. Proc Natl Acad Sci U S A 2011; 108: 1579-84. [CrossRef]

16. Jiang Z, Chen J, Du X, Cheng H, Wang X, Dong C. IL-25 blockade inhibits metastasis in breast cancer. Protein Cell 2017; 8: 191-201.

17. Vannella KM, Ramalingam TR, Borthwick LA, Barron L, Hart KM, Thompson RW, et al. Combinatorial targeting of TSLP, IL-25, and IL33 in type 2 cytokine-driven inflammation and fibrosis. Sci Transl Med 2016; 8: 337ra65. [CrossRef]

18. Luo Y, Yang Z, Su L, Shan J, Xu H, Xu Y, et al. Non-CSCs nourish CSCs through interleukin-17E-mediated activation of NF-kappaB and JAK/STAT3 signaling in human hepatocellular carcinoma. Cancer Lett 2016; 375: 390-9. [CrossRef]

19. Feng J, Li L, Ou Z, Li O, Gong B, Zhao Z, et al. IL-25 stimulates M2 macrophage polarization and thereby promotes mitochondrial respiratory capacity and lipolysis in adipose tissues against obesity. Cell Mol Immunol 2018; 15: 493-505. [CrossRef] 
20. Huang 0, Niu Z, Tan J, Yang J, Liu Y, Ma H, et al. IL-25 Elicits Innate Lymphoid Cells and Multipotent Progenitor Type 2 Cells That Reduce Renal Ischemic/Reperfusion Injury. J Am Soc Nephrol 2015; 26: 2199-211. [CrossRef]

21. Mantani PT, Dunér P, Bengtsson E, Alm R, Ljungcrantz I, Söderberg I, et al. IL-25 inhibits atherosclerosis development in apolipoprotein E deficient mice. PLoS One 2015; 10: e0117255. [CrossRef]

22. Ye J, Wang M, Xu Y, Liu J, Jiang $H$, Wang Z, et al. Sestrins increase in patients with coronary artery disease and associate with the severity of coronary stenosis. Clin Chim Acta 2017; 472: 51-7. [CrossRef]

23. Yao X, Sun $Y$, Wang W, Sun Y. Interleukin (IL)-25: Pleiotropic roles in asthma. Respirology 2016; 21: 638-47. [CrossRef]

24. Liu D, Cao T, Wang N, Liu C, Ma N, Tu R, et al. IL-25 attenuates rheumatoid arthritis through suppression of Th17 immune responses in an IL-13-dependent manner. Sci Rep 2016; 6: 36002. [CrossRef]

25. Javan M, Seyfizadeh N, Aslani S, Farhoodi M, Babaloo Z. Molecular analysis of interleukin-25 exons 1 and 2 and its serum levels in Iranian patients with multiple sclerosis. Am J Clin Exp Immunol 2014; 3: 91-6.

26. Su J, Chen T, Ji XY, Liu C, Yadav PK, Wu R, et al. IL-25 downregulates Th1/Th17 immune response in an IL-10-dependent manner in inflammatory bowel disease. Inflamm Bowel Dis 2013; 19: 720-8.

27. Lee JU, Chang HS, Lee HJ, Bae DJ, Son JH, Park JS, et al. Association of interleukin-25 levels with development of aspirin induced respiratory diseases. Respir Med 2017; 123: 71-8. [CrossRef]

28. Nadi E, Arjipour M, Sharifi S, Zamani A. Assay of IL-22 and IL-25 in serum, whole blood, and peripheral blood mononuclear cell cultures of patients with severe asthma. Allergol Immunopathol (Madr) 2014; 42: 402-6. [CrossRef]

29. Hasegawa T, Uga H, Mori A, Kurata H. Increased serum IL-17A and Th2 cytokine levels in patients with severe uncontrolled asthma. Eur Cytokine Netw 2017; 28: 8-18. [CrossRef]

30. Aalberse JA, van Thuijl $A 0$, Meijer $Y$, de Jager $W$, van der PalenMerkus T, Sprikkelman AB, et al. Plasma IL-25 is elevated in a subgroup of patients with clinical reactivity to peanut. Clin Transl Allergy 2013; 3: 40. [CrossRef]

31. Lavocat F, Ndongo-Thiam N, Miossec P. Interleukin-25 Produced by Synoviocytes Has Anti-inflammatory Effects by Acting As a Receptor Antagonist for Interleukin-17A Function. Front Immunol 2017; 8: 647.

32. Terrier B, Bieche I, Maisonobe T, Laurendeau I, Rosenzwajg M, Kahn $\mathrm{JE}$, et al. Interleukin-25: a cytokine linking eosinophils and adaptive immunity in Churg-Strauss syndrome. Blood 2010; 116: 4523-31.

33. Fort MM, Cheung J, Yen D, Li J, Zurawski SM, Lo S, et al. IL-25 induces IL-4, IL-5, and IL-13 and Th2-associated pathologies in vivo. Immunity 2001; 15: 985-95. [CrossRef]
34. Song X, Qian Y. IL-17 family cytokines mediated signaling in the pathogenesis of inflammatory diseases. Cell Signal 2013; 25: 233547. [CrossRef]

35. Andreakos E, Papadopoulos NG. IL-25: the missing link between allergy, viral infection, and asthma? Sci Transl Med 2014; 6: $256 f s 38$.

36. Ikeda K, Nakajima H, Suzuki K, Kagami S, Hirose K, Suto A, et al. Mast cells produce interleukin-25 upon Fc epsilon RI-mediated activation. Blood 2003; 101: 3594-6. [CrossRef]

37. Kang CM, Jang AS, Ahn MH, Shin JA, Kim JH, Choi YS, et al. Interleukin-25 and interleukin-13 production by alveolar macrophages in response to particles. Am J Respir Cell Mol Biol 2005; 33: 290-6.

38. Wang YH, Angkasekwinai P, Lu N, Voo KS, Arima K, Hanabuchi S, et al. IL-25 augments type 2 immune responses by enhancing the expansion and functions of TSLP-DC-activated Th2 memory cells. J Exp Med 2007; 204: 1837-47. [CrossRef]

39. Corrigan CJ, Wang W, Meng 0, Fang C, Eid G, Caballero MR, et al. Allergen-induced expression of IL-25 and IL-25 receptor in atopic asthmatic airways and late-phase cutaneous responses. J Allergy Clin Immunol 2011; 128: 116-24. [CrossRef]

40. Libby P, Ridker PM, Hansson GK. Progress and challenges in translating the biology of atherosclerosis. Nature 2011; 473: 317-25.

41. Van Dyke TE, van Winkelhoff AJ. Infection and inflammatory mechanisms. J Clin Periodontol 2013; 40 Suppl 14: S1-7. [CrossRef]

42. Kampits C, Montenegro MM, Ribeiro IW, Furtado MV, Polanczyk $\mathrm{CA}$, Rösing CK, et al. Periodontal disease and inflammatory blood cytokines in patients with stable coronary artery disease. J Appl Oral Sci 2016; 24: 352-8. [CrossRef]

43. Min X, Lu M, Tu S, Wang X, Zhou C, Wang S, et al. Serum Cytokine Profile in Relation to the Severity of Coronary Artery Disease. Biomed Res Int 2017; 2017: 4013685. [CrossRef]

44. Kumari N, Dwarakanath BS, Das A, Bhatt AN. Role of interleukin-6 in cancer progression and therapeutic resistance. Tumour Biol 2016; 37: 11553-72. [CrossRef]

45. Kleinschek MA, Owyang AM, Joyce-Shaikh $B$, Langrish $C L$, Chen $Y$, Gorman DM, et al. IL-25 regulates Th17 function in autoimmune inflammation. J Exp Med 2007; 204: 161-70. [CrossRef]

46. Suto H, Nambu A, Morita H, Yamaguchi S, Numata T, Yoshizaki T, et al. IL-25 enhances TH17 cell-mediated contact dermatitis by promoting IL-1 beta production by dermal dendritic cells. J Allergy Clin Immunol 2018; 142: 1500-9. [CrossRef]

47. Mantani PT, Duner P, Bengtsson E, Ljungcrantz I, Sundius L, To F, et al. Interleukin-25 (IL-25) has a protective role in atherosclerosis development in the aortic arch in mice. J Biol Chem 2018; 293: 6791801. [CrossRef] 